in tissue (on frozen tissue section or paraffin embedded) by PCR analysis of immunoglobulin V-D-J genes heavy chain rearrangements (FR1, FR2, FR3); monoclonal secretion was tested in serum protein electrophoresis.

Results: 40 patients with IgG4-ROD had orbital lesions at the onset of the disease and 13 developed them after 2 - 9 years from the onset. The frequency of different orbital anatomic structures involvement see in table 1.15 (28\%) patients had isolated orbital lesions while $38(72 \%)$ had systemic lgG4-RD with simultaneous involvement of 2 - 7 other organs. $6(14 \%)$ patients had unilateral orbital lesions. $23(53 \%)$ patients with IgG4-ROD had associated IgG4-related sialoadenitis and nasal lesions. Most common pathomorphological features of lgG4-ROD see in table 2. The most common laboratory features of IgG4-ROD were elevation of: total serum IgG (44\%), serum IgG4 (88\%), serum $\operatorname{lgE}(61 \%)$ and monoclonal serum secretion $(23 \%)$

Table 1. Prevalence of different orbital anatomic structures affection in IgG4-ROD ( $n=43$ )

\begin{tabular}{|l|l|}
\hline Anatomicstructure & $\mathrm{N}, \%$ \\
\hline Lacrimal gland & 93 \\
\hline Extraocular muscules & 85,5 \\
\hline Fibroinflammatory lesions of eyelids & 32,5 \\
\hline Retrobulbar infiltration & 32,5 \\
\hline Optic nerve thickening & 32,5 \\
Trigeminal involvement & 21 \\
\hline Nasolacrimal duct & 4,5 \\
Table 2. Pathomorphological features in IgG4-ROD (n=43)
\end{tabular}

\section{Pathomorphological feature}

\begin{tabular}{|l|l|}
\hline Pathom orphological feature & $\mathrm{N}, \%$ \\
\hline Lymphoplasmatic infiltrate & 93 \\
\hline Dense fibrosis & 93 \\
\hline Lymphoid follici formation & 69 \\
\hline Tissue eosinophilia & 32,5 \\
Obliterative phl ebitis & 14 \\
\hline Vasculitis & 7 \\
\hline IgG4/IgG $>40 \%$ & 90,5 \\
\hline MALT-lymphoma of lacrimal gland & 2,3 \\
\hline AL-amyloidosis & 2,3 \\
\hline
\end{tabular}

Conclusions: In our cohort of IgG4-RD patients orbit is the leading site of the disease $(64.5 \%$ of patients) and the disease onset ( $75 \%$ of patients). The majority of patients have bilateral orbital lesions (89\%) and systemic course of the disease with other organ involvement (72\%). Most often affected orbital anatomicstructures are lacrimal glands, extraocular muscles, retrobulbar infiltration with optic nerve thickening and fibroinflammatory lesions of eyelids. MALT-lymphoma of lacrimal gland and local AL-amyloidosis can complicate the long history of IgG4-ROD. Monoclonal serum secretion and B-cell clonality in the tissue in $23 \%$ of patients can potentially act as a background for lymphoma formation.

References:

[1] Uchida K, Masamune A, Shimosegawa T et al. Prevalence of IgG4-related disease in Japan based on nationwide survey in 2009. IntJ.Rheumatol. 2012;2012:358371.

[2] Umehara H, Okazaki K, Masaki $\mathrm{Y}$ et al. A novel clinical entity, IgG4-related disease (IgG4RD): general concept and details. ModRheumatol. 2012; 22 : $1-14$.

[3] Stone JH, Khosroshahi A, Deshpande V et al. Recommendations for the Nomenclature of IgG4-Related Disease and Its Individual Organ System Manifestations. Arth.Rheum. 2012; 64:3061-3067.

Disclosure of Interest: None declared

DOI: 10.1136/annrheumdis-2017-eular.4283

\section{FRI0622 A RETROSPECTIVE COHORT STUDY ABOUT THE CLINICAL CHARACTERISTICS OF IGG4-RELATED DISEASES}

X. Wu, C. Wang, Y. Miao, Y. Huang, L. Dong. Department of Rheumatology and Immunology, Tongji Hospital of Tongji Medical College, Huazhong University of Science and Technology, Wuhan, Hubei, China

Background: Immunoglobulin (Ig) G4-related disease (IgG4-RD) is an increasingly recognized systemic autoimmune disease in the past decade, and encompasses a wide spectrum of disorders previously considered to be unrelated, but with common pathologic, serologic, and clinical features. There have been several hundred literatures on IgG4-RD published in the past few years, however, we still cannot well characterize this complicated disease, including clinical features, treatments as well as mechanisms, which promotesus to further describe this disease.

Objectives: To describe the clinical presentations, laboratory features, imaging manifestations, treatments and evolutions in a cohort of 34 Chinese patients with immunoglobulin G-related diseases (IgG4-RD).

Methods: A retrospective study was performed in Wuhan Tongji Hospital. Electronically stored records of clinical, laboratory, imaging, and histological features were reviewed. The diagnoses were made according to comprehensive diagnostic criteria for IgG4-RD. Disease activity was assessed by IgG4-RD Responder Index (RI).This study was approved by the Ethical Committee of TongjiHospital (TJ-C20150603). All patients were contacted, and consents were obtained from all the participants.

Results: We found that among the whole 34 patients enrolled in this study (median age $=51$ years, male/female ratio $=1.4 / 1$ ), $65.2 \%$ tested patients had a declined HDL level, $82.3 \%$ tested patients had an elevated IgG4 level, and lymph node was the most frequently involved organ. 18F-FDG PET/CT had high sensitivity in providing information about the organ distribution of IgG4-RD. The IgG4 level and IgG4-RD RI in the multi-organs affected patients were significantly higher than that in the localized ones. Most patients were prescribed with glucocorticoids (GCs) alone or combined with disease modifying antirheumatic drugs (DMARDs), the majorities responded well, while some relapsed. Patients given rituximab (RTX) obtained good responses.

Table 1. The overall prognosis of 19 patients who were followed up

\begin{tabular}{|c|c|c|c|c|c|c|c|}
\hline Prognosis & GCs & $\begin{array}{c}\text { GCs } \\
+ \text { DMARDs }\end{array}$ & $\begin{array}{c}\text { Surgery } \\
+ \text { GCs }\end{array}$ & $\begin{aligned} & \text { Surgery } \\
&+ \text { DMARDs } \\
&\end{aligned}$ & $\begin{array}{c}\text { Antifungal } \\
\text { therapy }\end{array}$ & MMF & $\begin{array}{c}\text { Total, } \\
\mathrm{n} \\
\end{array}$ \\
\hline Remission, $\mathrm{n}$ & 5 & 1 & 2 & 0 & 0 & 1 & 9 \\
\hline Recurrence, $\mathrm{n}$ & 3 & 4 & 0 & 0 & 0 & 0 & 7 \\
\hline Worsen/Persistence, $\mathrm{n}$ & 0 & 1 & 0 & 1 & 1 & 0 & 3 \\
\hline Total, $\mathrm{n}$ & 8 & 6 & 2 & 1 & 1 & 1 & 19 \\
\hline
\end{tabular}

Total, $\mathrm{n}$

Abbreviations: GCs, glucocorticoids; DMARDs, disease-modifying anti-rheumatic drugs; MMF, mycophenolate mofetil.
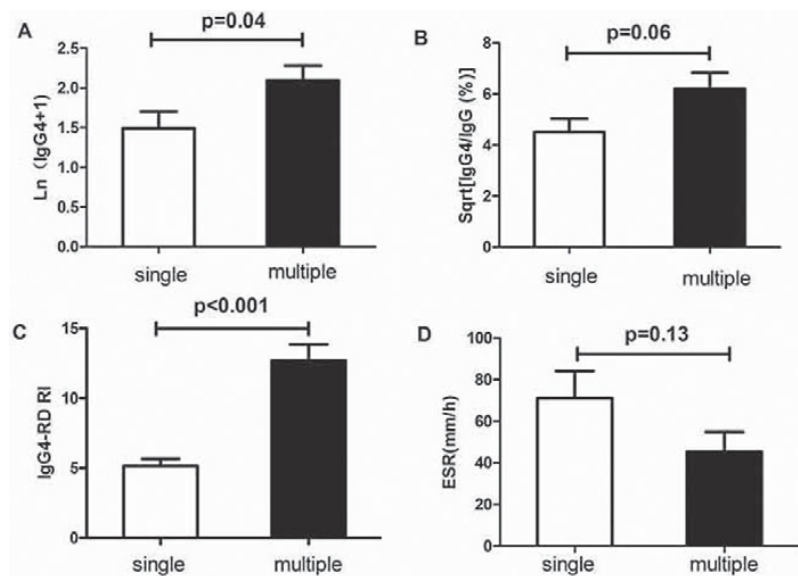

Fig 1: Analysis of $\operatorname{lgG} 4, \lg G 4 / \lg G(\%), \lg G 4-R D$ RI, and ESR levels between patients of single organ involvement and multiple organs involvement.

Conclusions: It was concluded that 18F-FDG PET/CT can be an effective tool to detect involved lesions of IgG4-RD. The addition of DMARDs to the initial GCs therapy in the remission induction stage may not bring much benefit. The most appropriate dosage schedule of RTX needs to be further investigated. Patients with younger age, longer duration of disease, higher IgG4 level and IgG4/lgG ratio may more commonly experience recurrence.

References:

[1] Hamano H, Kawa S, Horiuchi $A$, et al. High serum IgG4 concentrations in patients with sclerosing pancreatitis. N Engl J Med, 2001,344(10):732-738.

[2] Islam AD, Selmi $C$, Datta-Mitra $A$, et al. The changing faces of IgG4related disease: Clinical manifestations and pathogenesis. Autoimmun Rev, 2015,14(10):914-922.

[3] Martinez-de-Alegria A, Baleato-Gonzalez S, Garcia-Figueiras R, et al. IgG4related Disease from Head to Toe. Radiographics, 2015,35(7):2007-2025.

Disclosure of Interest: None declared

DOI: 10.1136/annrheumdis-2017-eular.3623

\section{FRIDAY, 16 JUNE 2017}

\section{Diagnostics and imaging procedures}

\section{FRI0623 [18F]FLUORIDE PET-CT IMAGING OF BONE FORMATION IN ANKYLOSING SPONDYLITIS BEFORE AND AFTER 12 WEEKS OF ANTI-TNF TREATMENT}

S. Bruijnen ${ }^{1}$, N. Verweij ${ }^{1}$, L. van Duivenvoorde ${ }^{1}$, N. Bravenboer ${ }^{2}$, D. Baeten ${ }^{1}$ C. van Denderen ${ }^{1}$, I. van der Horst-Bruinsma ${ }^{1}$, A. Voskuyl ${ }^{1}$, P. van de Ven ${ }^{3}$, J. Bot ${ }^{4}$, B. Boden ${ }^{4}$, A. Lammertsma ${ }^{4}$, O. Hoekstra ${ }^{4}$, P. Raijmakers ${ }^{4}$,

C. van der Laken ${ }^{1}$. ${ }^{1}$ Rheumatology, Amsterdam Rheumatology and immunology Center; ${ }^{2}$ Clinical Chemistry; ${ }^{3}$ Epidemiology and Biostatistics; ${ }^{4}$ Radiology \& Nuclear Medicine, VU University Medical Center, Amsterdam, Netherlands

Background: Excessive bone formation is an important hallmark of ankylosing spondylitis (AS). Recently, it has been demonstrated that axial bony lesions in AS patients can be visualized using $\left[{ }^{18} \mathrm{~F}\right]$ fluoride PET-CT. This technique may also provide opportunities to monitor changes in bone formation in axial AS lesions during therapy.

Objectives: To assess (1)changes in $\left[{ }^{18} \mathrm{~F}\right]$ fluoride uptake in axial lesions of AS patients before and 12 weeks after treatment with TNF-inhibitors (TNFi) and (2) whether $\left[{ }^{18} \mathrm{~F}\right]$ fluoride uptake on PET is related to focal bone formation in spine biopsies. 
Methods: Twelve TNFi naive AS patients (female 7/12; age 39 \pm 11 years) with high disease activity (BASDAI $5.5 \pm 1.1$ ) were included. $\left[{ }^{18} \mathrm{~F}\right]$ fluoride PET-CT scans were performed before initiation of TNFi therapy. In 2 patients, biopsies were obtained from PET identified spine lesions for histologic analysis. Of the remaining 10 patients, a second $\left[{ }^{18} \mathrm{~F}\right]$ fluoride PET-CT scan was performed after 12 weeks of TNFi treatment. PET scans were scored visually for positivity by two blinded expert readers. Subsequently, $\left[{ }^{18} \mathrm{~F}\right]$ fluoride uptake was quantified in PET positive $\left(\mathrm{PET}^{+}\right)$lesions using the standardized uptake value corrected for individual integrated whole blood activity concentrations $\left(S_{U} U V_{A U C}\right)$. Clinical response to TNFi was defined according to ASAS20 at 24 weeks.

Results: At baseline, in all patients at least one axial $\mathrm{PET}^{+}$lesion was found. In spine, 6/10 patients showed 84 lesions (range 2-30;63\% thoracic spine, primarily costovertebral joints) and in the $\mathrm{SI}$ joints in $9 / 10$ patients were $\mathrm{PET}^{+}$ (Fig A). Histological analysis of PET+ lesions in the spine confirmed local osteoid formation, which was nearly absent in PET negative lesions. Quantitative PET analysis revealed significantly lower $\left[{ }^{18} \mathrm{~F}\right]$ fluoride uptake in spine lesions at baseline in responders than in non-responders. This difference remained after 12 weeks of treatment (mean difference in SUV Auc : $-0.5,95 \% \mathrm{Cl}:[-$ $0.7,-0.2], p=0.001)$. After 12 weeks of TNFi treatment, $\left[{ }^{18} \mathrm{~F}\right]$ fluoride uptake in clinical responders decreased significantly in the costovertebral (mean difference

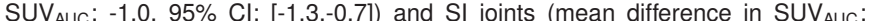
$-1.2,95 \% \mathrm{Cl}:[-2.3,-0.2]$ ) (fig B) in contrast to non-responders (mean difference in SUV ${ }_{\text {AUC }}:-0.4,95 \% \mathrm{Cl}:[-2.3,1.6]$ and $+0.4,95 \% \mathrm{Cl}$ : $[-0.6,1.4]$, respectively). $\left[{ }^{18} \mathrm{~F}\right]$ fluoride uptake in other spinal lesions such as bridging syndesmophytes showed heterogeneous response without a significant decrease in $\left[{ }^{18} \mathrm{~F}\right]$ fluoride accumulation over time at a group level.

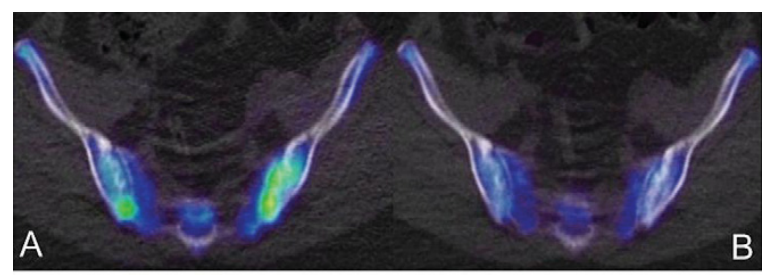

Figure: Example of $\left[{ }^{18} \mathrm{~F}\right]$ fluoride uptake in both $\mathrm{SI}$ joints at baseline $(\mathrm{A})$ and a clear decrease of uptake after 12 weeks of TNF-I (B).

Conclusions: $\left[{ }^{18} \mathrm{~F}\right]$ fluoride PET-CT enables non-invasive visualization of (changes in) lesions with bone formation of the whole spine and SI joints in clinically active AS patients, which is confirmed by histological signs of osteoid formation. Part of these lesions, in particular costovertebral lesions in spine and SI joints, decreased in clinical responders to TNFi (and not in non-responders), whereas other spinal lesions remained unchanged at a group level.

Disclosure of Interest: None declared

DOI: 10.1136/annrheumdis-2017-eular.2004

\section{FRI0624 STRUCTURAL MRI-BASED CONNECTOMICS IN SLE: A PILOT STUDY}

G.A. Ramirez ${ }^{1,2}$, E.P. Bozzolo ${ }^{1}$, P. Preziosa ${ }^{3}$, M.A. Rocca ${ }^{3}$, L. Moiola ${ }^{3}$ L.A. Coletto ${ }^{2}$, E. Tombetti ${ }^{1,2}$, P. Rovere-Querini ${ }^{1,2}$, M. Filippi ${ }^{2,3}$

A.A. Manfredi ${ }^{1,2}{ }^{1}{ }^{1}$ Unit of Allergy, Immunology, Rheumatology and Rare

Diseases, IRCCS Ospedale San Raffaele; ${ }^{2}$ Università Vita-Salute San Raffaele ${ }^{3}$ Institute of Experimental Neurology and Unit of Neurology, IRCCS Ospedale San Raffaele, Milano, Italy

Background: Neuropsychiatric manifestations are common in patients with systemic lupus erythematosus (SLE). Furthermore, subclinical brain damage occurs in an even higher fraction of patients. However, little is known about the effect of these phenomena on brain connectivity. MRI-based connectomics relies on graph analysis of structural and functional images to detect alterations of the topographic organization of the brain and has been successfully employed to dissect network disassembly in neuroinflammatory diseases such as multiple sclerosis (MS) and Devic's syndrome.

Objectives: To investigate the topographic organization of the brain of patients with SLE with and without neuropsychiatric manifestations.

Methods: Thirty-two patients with SLE (12 with overt neuropsychiatric involvement as per ACR criteria) were enrolled and compared with 32 healthy controls (HC) and 32 patients with relapsing-remitting MS, all matched for sex, age and disease duration (where applicable). Diffusion tensor (DT) and dual-echo MRI scans were obtained. Structural connectivity matrices between 116 cortical and subcortical brain regions were estimated and global and nodal network metrics were calculated.

Results: Conventional MRI revealed that patients with SLE had significantly higher T2-lesional volumes when compared to controls $(p<0.0001)$. Patients with definite NPSLE had a higher lesion burden $(\mathrm{p}=0.006)$. Network strength, transitivity and global efficiency were all significantly impaired in patients with MS and SLE when compared to HC $(p<0.0001)$. MS and SLE were also characterized by higher average path length when compared to $\mathrm{HC}(p<0.0001)$. Global structural alterations were more significant in MS patients than in patients with SLE ( $p$ from 0.005 to 0.01 at multiple comparison). However, antiDNA-positive patients $(n=24)$ showed a more severe phenotype when compared to antiDNA-negative patients ( $p$ from 0.026 to 0.041 ) and did not differ significantly from patients with MS. When regional hubs were analysed, patients with SLE and MS showed a reduced strength compared to $\mathrm{HC}$ ( $\mathrm{p}$ from $<0.0001$ to 0.001 at multiple comparison). Hub strength impairment was more pronounced in MS when compared to SLE and preferentially involved hubs located in fronto-temporo-parieto-occipital cortices, subcortical nuclei (including the thalamus, caudate nucleus and putamen) and cerebellum ( $\mathrm{p}$ from 0.001 to 0.05 at multiple comparison). No significant associations were found between global structural parameters, clinical diagnosis of neuropsychiatric SLE, other SLE sub-phenotypes, presence of antiphospholipid antibodies, antiphospholipid syndrome and SLE-related damage burden.

Conclusions: Structural alterations of global and regional brain connectivity occur in patients with SLE, irrespectively of the clinical phenotype. AntiDNA-positive patients are characterized by a more severe phenotype, which is similar to that of patients with relapsing-remitting MS.

References:

[1] Sabbadini MG et al, Lupus, 1999.

[2] Filippi M et al., Lancet Neurol, 2013.

[3] Rocca MA et al., Brain Struct Funct, 2016.

Disclosure of Interest: None declared

DOI: 10.1136/annrheumdis-2017-eular.4264

\section{FRI0625 IMPROVEMENT OF JOINT INFLAMMATION AS ASSESSED BY MRI AND POWER DOPPLER ULTRASOUND (PDUS) IN AN OPEN LABEL STUDY IN PATIENTS WITH ACTIVE PSORIATIC ARTHRITIS TREATED WITH SECUKINUMAB (PSARTROS)}

E. Kampylafka, I. D'Oliveira, C. Linz, V. Lerchen, M. Englbrecht, A. Kleyer, J. Rech, G. Schett, A.J. Hueber. Department of Internal Medicine 3 Rheumatology and Immunology, Friedrich-Alexander-University

Erlangen-Nürnberg (FAU) and Universitätsklinikum Erlangen, Ulmenweg 18 , 91054 Erlangen, Germany, Erlangen, Germany

Background: Secukinumab, an anti-interleukin 17A monoclonal antibody, showed significant improvement of signs and symptoms of psoriatic arthritis (PsA) in FUTURE 1 study. Available studies used conventional radiography, not allowing a deeper imaging analysis of the inflammatory changes during application.

Objectives: To assess short term efficacy of secukinumab on inflammation and structural damage according to change in OMERACT-EULAR ultrasound score and the MRI PsAMRIS score in PsA patients.

Methods: PsA patients with active disease (TJC and SJC $>3$ ), were included in the 24 week open label prospective PSARTROS study and treated with subcutaneous secukinumab $300 \mathrm{mg}$ once weekly over 4 weeks, then once every 4 weeks. Baseline 1,5T MRI hand scans and ultrasound imaging of 28 joints were performed at baseline and after 24 weeks of treatment. MRI was scored according to PsAMRIS, ultrasound for synovial hypertrophy and Doppler activity according to OMERACT scores. Statistical significance was set at $\mathrm{p}<0.05$.

Results: 20 patients, mean age $52 \pm 9.9$ years, $60 \%$ female, mean disease duration $6.7 \pm 5.9$ years, $50 \%$ naïve for biological therapy, were included in the study. Three patients were early discontinued (recurrent pharyngitis, lack of efficacy, withdrawal of consent), and were not included into the longitudinal analysis. Baseline DAS28 was $5.03 \pm 0.96$, baseline DAPSA was $32.2 \pm 12$. 1 . On baseline MRI, all patients had at least one inflammatory sign (synovitis: $90 \%$, osteitis: $20 \%$, periarticular inflammation: $25 \%$, flexor tenosynovitis: $35 \%$, bone proliferation: $30 \%$, erosions: $60 \%$ ). Baseline composite PsAMRIS score

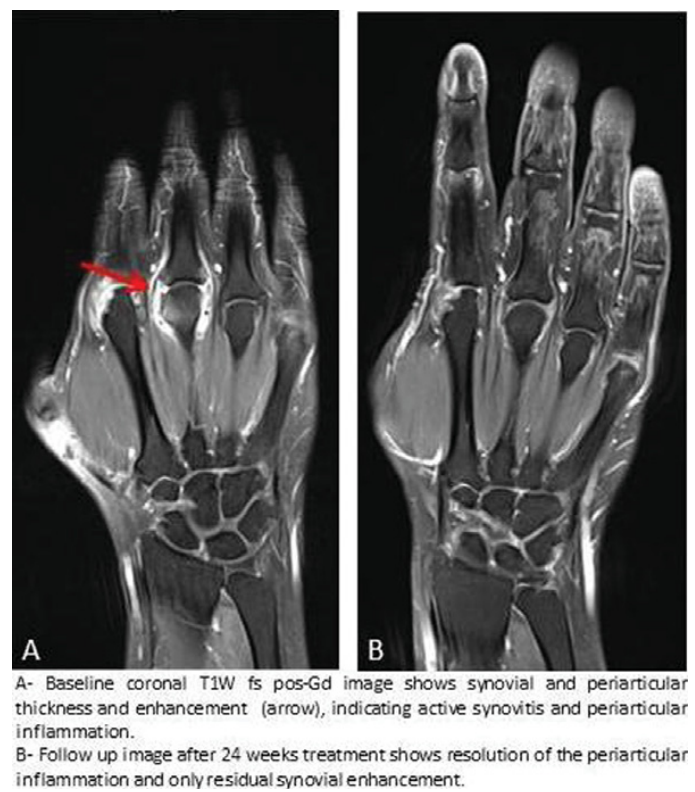

